Trauma Berufskrankh 2015 • 17[Suppl 1]:74-77 DOI 10.1007/s10039-014-2118-y

Online publiziert: 22. Januar 2015

c) Springer-Verlag Berlin Heidelberg 2015

\author{
D. Schneidmueller ${ }^{1} \cdot$ I. Marzi ${ }^{2} \cdot$ V. Bühren ${ }^{1}$ \\ ${ }^{1}$ Abteilung für Unfallchirurgie, Sportorthopädie und Kindertraumatologie der BGU Murnau, \\ Klinikum Garmisch-Partenkirchen, Murnau \\ 2 Klinik für Unfall-, Hand- und Wiederherstellungschirurgie, \\ Goethe-Universität Frankfurt am Main, Frankfurt am Main
}

\title{
Sturz auf den Ellenbogen
}

\section{Diagnostik von Ellenbogenverletzungen}

Ellenbogenverletzungen bei Kindern und Jugendlichen stellen für die behandelnden Ärzte häufig eine Herausforderung dar. Nicht selten werden gerade in dieser Lokalisation Frakturen übersehen [1, 3, 11, 14]. Pseudarthrosen, Wachstumsstörungen und Deformitäten können hier zu relevanten Langzeitfolgen führen [13].

Ziel der Untersuchung muss daher sein, eine aktiv behandlungsbedürftige Verletzung auszuschließen. Hierzu gehören alle dislozierten Frakturen außerhalb der Grenzen der Spontankorrektur sowie alle nicht oder wenig dislozierten Frakturen, die trotz symptomatischer Therapie mittels Immobilisation bei Nichterkennen zu Problemen führen können.

\section{Bildgebung}

\section{Konventionelles Röntgen}

Grundlage der Diagnostik stellt nach wie vor das konventionelle Röntgenbild in 2 Ebenen dar. Hierbei soll auch im Sinne der Strahlenhygiene nicht auf die 2. Ebene verzichtet werden, da wenig dislozierte Frakturen manchmal nur in einer Ebene erkennbar sind. Eine Ausnahme stellen vollständig dislozierte Frakturen dar, bei denen eine klare Operationsindikation besteht. Hier liefert eine 2. Ebene keine relevanten Zusatzinformationen. Sie kann schmerzfrei in Narkose nachgeholt werden.

Ebenso sollte durch eine klinische Untersuchung die Verletzungsregion eingegrenzt werden, um gezielte Röntgenaufnahmen anfertigen zu können. Sog. Übersichtsaufnahmen von z. B. einer Extremi- tät in einer Ebene sollten vermieden werden.

Die Vielzahl und die Varianz der Knochenkerne am noch überwiegend knorpelig angelegten Ellenbogengelenk erschweren die Beurteilung der Röntgenbilder. Das Auftreten der Knochenkerne erfolgt in bestimmten Alterszeiträumen, die bei der Befundung einer Aufnahme bekannt sein müssen (• Abb. 1).

Ein indirektes Zeichen für das Vorliegen einer Verletzung ist das sog. positive Fettkörperzeichen („fad pad sign“; [10, 15]): Normalerweise verschwindet der am Humerusschaft anliegende Fettkörper in der Fossa coronoidea und der Fossa olecrani. Bei Vorliegen eines intraartikulären Ergusses wird dieser zwar intraartikulär, jedoch extrasynovial liegende Fettkörper vom Humerusschaft abgehoben, was dann als positives Fettkörperzeichen auf dem konventionellen Röntgenbild zu sehen ist (- Abb. 2). Ist dieses Zeichen nicht vorhanden, ist die Wahrscheinlichkeit für das Vorliegen einer relevanten Verletzung gering [4].

Die auch heute immer wieder angeführte Vergleichsaufnahme der Gegenseite ist nicht von Nutzen. Mehrere Studien haben gezeigt, dass sie nicht die Kenntnis der Anatomie sowie der möglichen Verletzungsmuster ersetzt [6]. Damit spielt sie heutzutage in der Akutdiagnostik von Verletzungen keine Rolle mehr.

\section{Sonographie}

Ihre Vorteile sind die fehlende Strahlenbelastung, die schnelle Durchführbarkeit und die vielfältige Verfügbarkeit. Al- lerdings ist sie noch wenig standardisiert, und die Ergebnisse sind stark vom Untersucher abhängig. Noch ersetzt sie das Röntgenbild nicht. Ansätze gibt es jedoch

- in der Unterscheidung von vollständigen von den sog. hängenden Condylus-radialis-Frakturen,

- in der Verlaufsbeurteilung von Frakturen oder

- in der sonographischen Bestimmung des Rotationsfehlers der suprakondylären Humerusfraktur $[9,16]$.

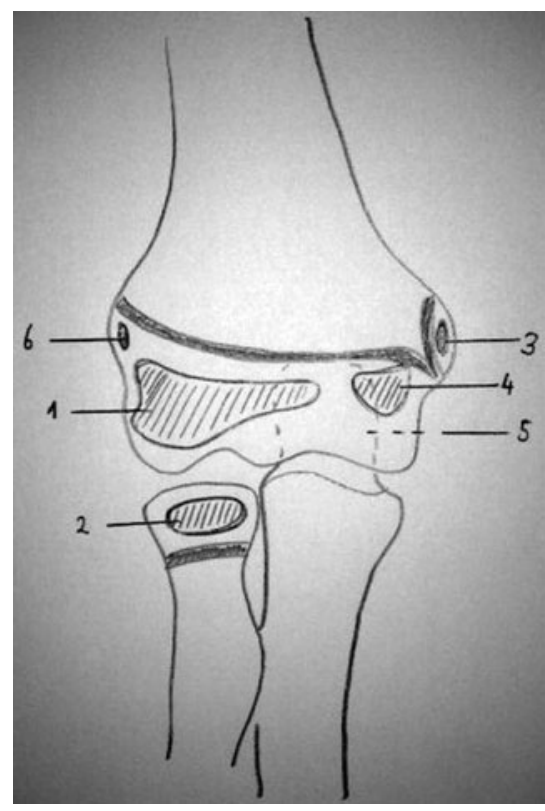

Abb. $1 \Delta$ Auftreten der Knochenkerne am Ellenbogengelenk, 1 Capitulum: 1. Lebensjahr, 2 Radiusköpfchen: 3 . bis 5. Lebensjahr, 3 Epicondylus ulnaris: 4. bis 5. Lebensjahr, 4 Trochlea: 8 . bis 10. Lebensjahr, 5 Olekranon: 8. bis 9 . Lebensjahr, 6 Epicondylus radialis: 10 . bis 11 . Lebensjahr 

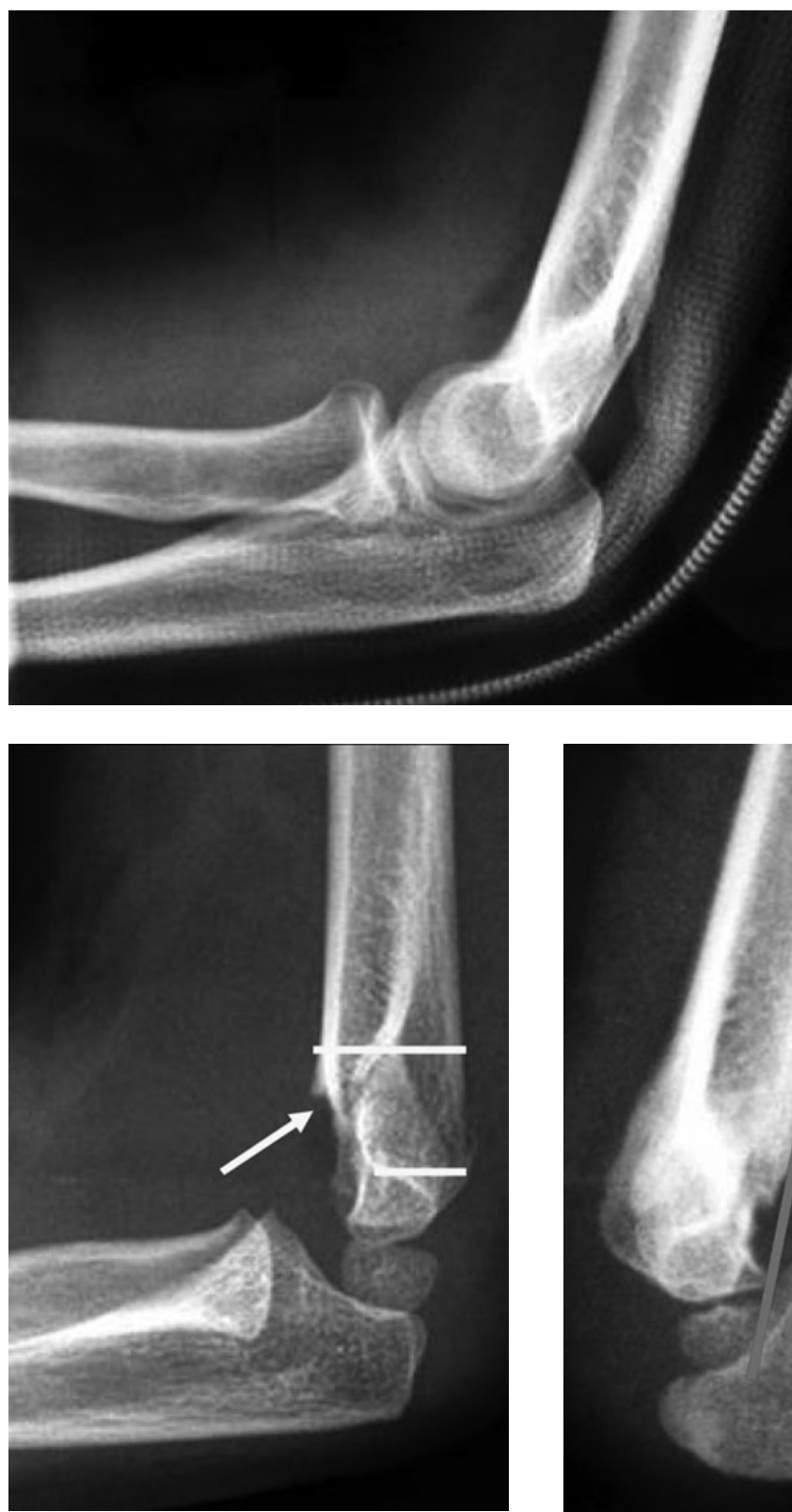

Abb. $4 \Delta$ Rotationsfehlstellung einer suprakondylären Humerusfraktur im sagittalen Röntgenbild mit ventralem Rotationssporn (Pfeil) sowie Kalibersprung der Fragmente

\section{Magnetresonanz- tomographie (MRT)}

Bei stetig verbesserter Qualität und zunehmenden Einsatzmöglichkeiten gewinnt sie auch am Ellenbogengelenk an Bedeutung (• Abb. 3).

Die therapeutische Relevanz der durch die MRT gewonnenen Informationen wird jedoch kontrovers diskutiert [2, 10, 12]: Griffith et al. [2] konnten in einer
Abb. $2<$ Positives Fettkörperzeichen als Hinweis auf das Vorliegen eines relevanten intraartikulären Ergusses

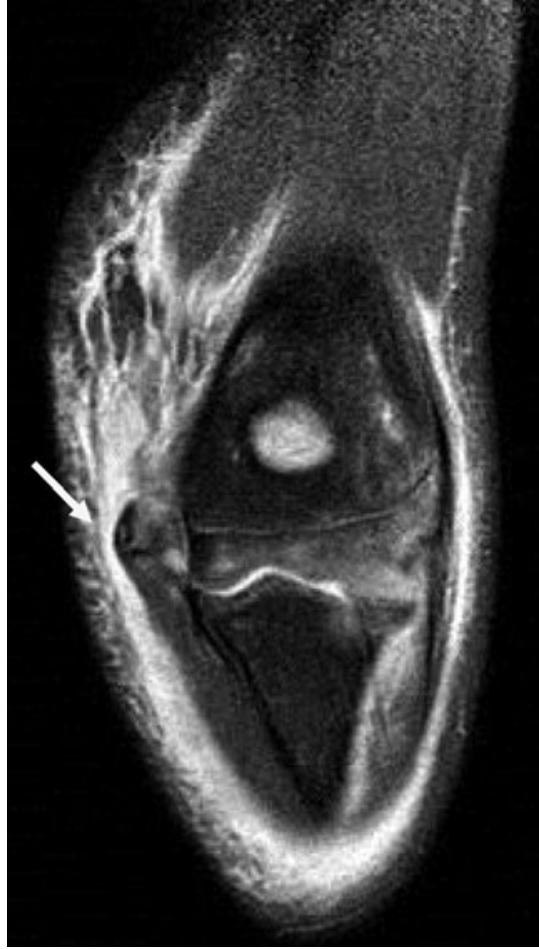

Abb. $3 \Delta$ MRT mit knöchernem Seitenbandausriss am Ellenbogen

\section{Computertomographie (CT)}

Sie spielt in der Routinediagnostik bei Kindern eine untergeordnete Rolle. Der Großteil des Ellenbogengelenks ist noch nicht mineralisiert und durch Röntgenstrahlung nicht adäquat darstellbar. Zudem sind komplexe Gelenkfrakturen bei Kindern eine Seltenheit. Im Adoleszentenalter können solche seltenen Gelenkfrakturen im Sinne z. B. einer Y-Fraktur des distalen Humerus auftreten, bei denen u. U. eine CT zur besseren Darstellung indiziert ist. Ebenso kann sie bei posttraumatischen Fehlstellungen im Rahmen einer dreidimensionalen Rekonstruktion zur Beurteilung der Achsen und Gelenkstellungen sinnvoll sein.

Untersuchung an 50 Ellenbogengelenken keinen Einfluss durch die Zusatzinformationen der MRT auf die Therapie, die Behandlungsdauer oder das Outcome beobachten. Sie spielt weniger in der Akutdiagnostik als in der Beurteilung von prolongierten Heilverläufen eine Rolle. Hier können Knorpeldefekte, Osteonekrosen oder freie Gelenkkörper ausgeschlossen bzw. dargestellt werden.

\section{Verletzungsmuster}

Die Kenntnis der möglichen Verletzungsmuster ist Grundvoraussetzung, um diese auch im Röntgenbild zu diagnostizieren. Dabei gibt es für jede Altersgruppen eine typische Ellenbogenverletzung. Die häufigste Verletzung mit 55\% der Fälle ist die suprakondyläre Humerusfrakur mit einem Altersgipfel um das 6. Lebensjahr, 
gefolgt von der Condylus-radialis-Fraktur mit 19\% mit einem Altersgipfel bei den Kindergartenkindern um das 4. Lebensjahr. Der Epicondylus-ulnaris-Abriss (7\%) tritt häufig im Rahmen einer Ellenbogenluxation bei schon älteren Kindern mit einem Altersgipfel um das 11. Lebensjahr, die Radiushalsfraktur (9\%) um das 9. Lebensjahr und die Olekranonfraktur (10\%) um das 6. Lebensjahr auf [5].

Ziel ist es, eine aktiv behandlungsbedürftige Verletzung auszuschließen. Dazu gehören vollständig dislozierte Frakturen, die zumindest diagnostisch keine Schwierigkeit darstellen. Problematisch sind die nicht oder wenig dislozierten Frakturen, die bei Nichterkennen und rein symptomatischer Therapie zu Problemen führen können. Hierzu gehören im Wesentlichen die suprakondyläre Humerusfraktur, die Condylus-radialis-Fraktur und die Radiusköpfchenluxation $[8,7,17]$.

\section{Suprakondyläre Humerusfraktur}

Problematisch ist die Unterscheidung der stabilen von der instabilen Fraktur bei geringer Dislokation. Die Instabilität ist durch den sog. Rotationsfehler gekennzeichnet. Durch die spezielle Anatomie des distalen Humerus verkleinert sich die Auflagefläche der beiden Frakturfragmente durch Rotation deutlich, sodass es zu einem Abkippen meist in eine Varusfehlstellung kommen kann. Diese führt bei Ausheilung zu einem Cubitus varus mit v. a. kosmetischer Beeinträchtigung der Patienten.

Der Rotationsfehler ist im seitlichen Röntgenbild durch den sog. Rotationssporn sowie den Kalibersprung der beiden Fragmente gekennzeichnet (• Abb.4). Daneben muss auf eine Dislokation in der Sagittalebene geachtet werden. Hier ist die sog. Rogers-Hilfslinie nützlich (• Abb.5). Sie stellt eine Verlängerung der ventralen Humeruskortikalis dar, die im Normalfall das Capitulum humeri am Übergang vom mittleren zum dorsalen Drittel schneidet. Meist liegt eine Dislokation nach dorsal mit einer sog. Antekurvationsfehlstellung vor. Kommt es zu einer knöchernen Konsolidierung in dieser Position, können relevante Funktionseinschränkungen resultieren. (Die Antekurvationsfehlstellung führt zu einer ver-

Trauma Berufskrankh 2015 - 17[Suppl 1]:74-77 DOI 10.1007/s10039-014-2118-y

(c) Springer-Verlag Berlin Heidelberg 2015

D. Schneidmueller $\cdot$ I. Marzi $\cdot$ V. Bühren

Sturz auf den Ellenbogen.

Diagnostik von Ellenbogenverletzungen

\section{Zusammenfassung}

Hintergrund. Die Diagnostik von Ellenbogenverletzungen stellt eine besondere Herausforderung dar. Nicht selten werden hier relevante Verletzungen mit Langzeitfolgen übersehen.

Diagnostik. Grundlage der Diagnostik ist die Kenntnis der möglichen Verletzungsmuster sowie der alterstypischen Verteilung der einzelnen Verletzungen. Ziel ist es, in der Akutdiagnostik eine aktiv behandlungsbedürftige Verletzung zu erkennen bzw. auszuschließen. Dabei bildet das konventionelle Röntgenbild in 2 Ebenen nach wie vor die Basis der bildgebenden Diagnostik. Die Magnetresonanztomographie spielt häufig bei pro- longierten Heilverläufen zum Ausschluss z. B. einer okkulten Fraktur oder von Knorpelläsionen eine Rolle. Aufgrund der geringen Ossifikation beim Kind wird die Computertomographie i.d.R. erst beim Adoleszenten zur Beurteilung der seltenen Gelenkfraktur des distalen Humerus eingesetzt. Die Sonographie ermöglicht die Darstellung nichtossifizierter Anteile und von Frakturen, die im Röntgen nicht sichtbar sind.

\section{Schlüsselwörter}

Ellenbogen · Verletzungen · Kinder ·

Jugendliche · Röntgen

\section{Fall on the elbow. Diagnostics of elbow injuries}

\section{Abstract}

Background. The diagnostics of elbow injuries pose a particular challenge. Relevant injuries are misdiagnosed relatively often at this location with long-term consequences. Diagnostics. The diagnostics are based on knowledge of the possible injury patterns and the typical age distribution of the specific injuries. The goal is to identify injuries requiring an active intervention. The conventional anteroposterior and lateral radiographs constitute the basis of diagnostic imaging. Magnetic resonance imaging is helpful in cases of prolonged healing to exclude, for example an occult fracture or cartilage injuries. Due to the little ossification of the pediatric elbow computer tomography usually is used not until adolescence to evaluate the rare complex articular fractures of the distal humerus. Ultrasonography allows visualization of the cartilaginous parts of the elbow and occult fractures which are not visible in X-ray images.

\section{Keywords}

Elbow · Injuries · Children · Adolescents · X-ray besserten Überstreckbarkeit und zu einer reduzierten Beugefähigkeit). Eine Spontankorrektur dieser Fehlstellung ist nur um wenige Grad bei Kindern bis etwa zum 7. Lebensjahr möglich, sodass sie hier nur eingeschränkt miteingeplant werden kann.

\section{Condylus-radialis-Fraktur}

Sie tritt bei noch sehr jungen Kindern auf, bei denen die Epiphyse zum Großteil noch überwiegend knorpelig angelegt ist. Das macht die Unterscheidung einer vollständigen (instabilen) von einer unvollständigen bzw. sog. hängenden Fraktur (stabil) im konventionellen Röntgenbild schwierig ( $\bullet$ Abb. 6).
Übersieht man eine instabile vollständige Condylus-radialis-Fraktur, ist das Risiko einer Pseudarthrosenbildung bzw. eines radialen Überwachstums mit Cubitus-varus-Bildung bei verzögerter Frakturheilung hoch. Zudem handelt es sich um eine Gelenkfraktur, bei der Stufenbildungen der Gelenkfläche vermieden und eine anatomische Rekonstruktion angestrebt werden sollten. Möglichkeiten der primären Differenzierung liefern die Sonographie, die wenig standardisiert ist und viel Erfahrung benötigt, und die MRT. Letztere ist jedoch aufwendig, zeitund kostenintensiv, sodass die Indikation hierfür sorgfältig abgewogen werden sollte. Zudem wird bei den noch jungen Kindern zu ihrer Durchführung nicht selten eine Narkose notwendig. Eine einfache 


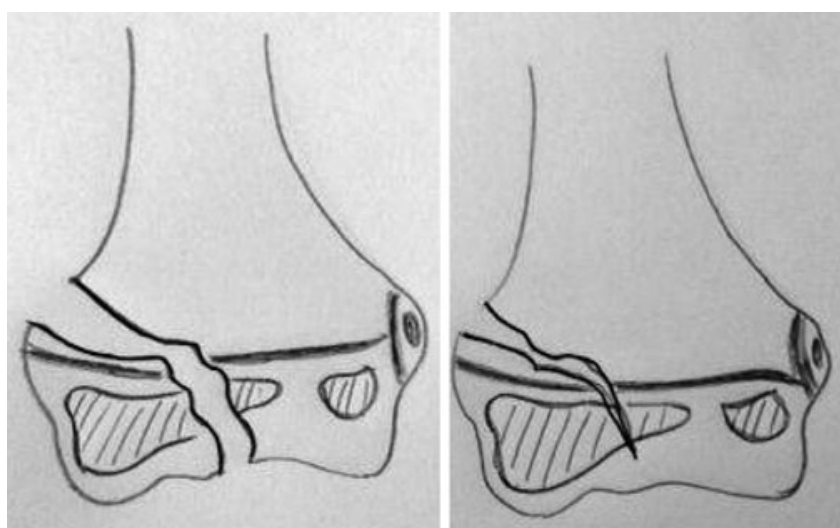

Abb. $6 \Delta$ Unterscheidung der vollständigen stabilen von der hängenden stabilen Condylus-radialis-Fraktur

Alternative sind bei vermeintlich stabilen, primär undislozierten Frakturen eine Immobilisation und nach 3 bis 5 Tagen die Durchführung einer gipsfreien Röntgenkontrolle zum Ausschluss einer Sekundärdislokation. Bleibt diese aus, kann man von einer stabilen Fraktur ausgehen und eine konservative Therapie anschließen.

\section{Radiusköpfchenluxation/ Monteggia-Fraktur}

Die Radiusköpfchenluxation ist die am häufigsten übersehene Verletzung am Ellenbogengelenk. Durch das fehlende Widerlager bei persistierender Luxation kommt es zu einer Überlänge und Verplumpung des Radius, sodass Funktionseinschränkungen, Beschwerden und Instabilitäten resultieren können. In jedem Röntgenbild des Ellenbogengelenks, egal welcher Projektion, muss sich die Verlängerung des Radiusköpfchens auf den Knochenkern des Capitulums projizieren (• Abb. 7). Liegt eine vermeintlich isolierte Luxation vor, muss immer eine Röntgenaufnahme des Unterarms zum Ausschluss einer Ulnapathologie im Rahmen einer Monteggia-Verletzung angeschlossen werden. Umgekehrt ist bei jeder Ulnaschaftfraktur eine Röntgenaufnahme des Ellenbogens zur Beurteilung der Stellung des Radiusköpfchens erforderlich.

\section{Fazit für die Praxis}

- Die Kenntnis der Entwicklung, Anatomie und möglichen Verletzungsmuster des Ellenbogengelenks ist Vo-

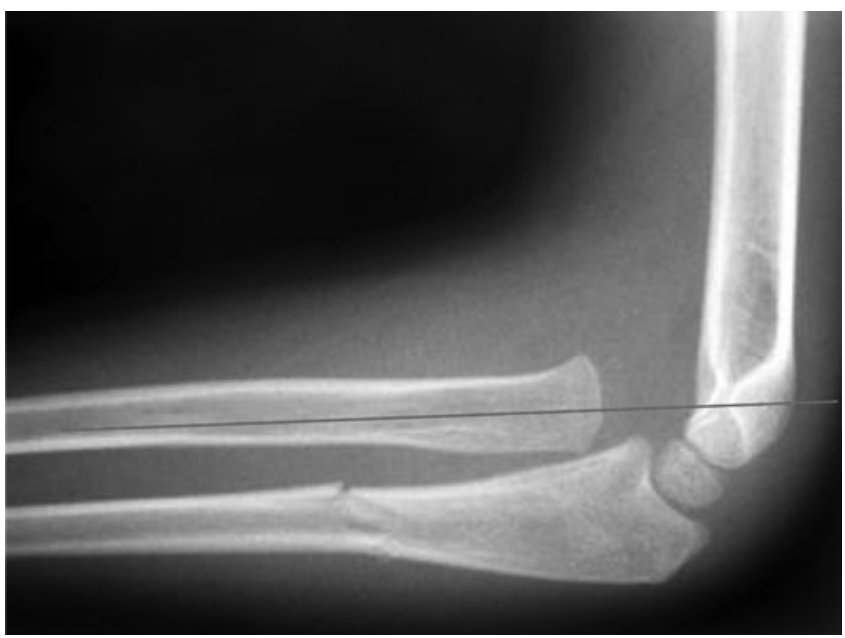

Abb. $7 \Delta$ Monteggia-Fraktur mit Fraktur der proximalen Ulna und Luxation des Radiusköpfchens nach ventral; Verlängerung des Radiusköpfchens projiziert sich nicht auf den Capitulumkern

raussetzung für die Beurteilung eines Röntgenbilds im Wachstumsalter.

- Bei der Diagnostik einer potenziellen Ellenbogenverletzung müssen alle aktiv behandlungsbedürftigen Verletzungen ausgeschlossen werden.

- Auch bei unauffälligem Röntgenbefund sollte entsprechend des klinischen Befunds eine symptomorientierte Therapie mittels Oberarmschiene erfolgen.

\section{Korrespondenzadresse}

PD Dr. D. Schneidmueller

Abteilung für Unfallchirurgie, Sportorthopädie und Kindertraumatologie der BGU Murnau, Klinikum Garmisch-Partenkirchen,

Professor-Küntscher-Str. 8, 82418 Murnau Dorien.Schneidmueller@bgu-murnau.de

\section{Einhaltung ethischer Richtlinien}

Interessenkonflikt. D. Schneidmueller, I. Marzi und V. Bühren geben an, dass kein Interessenkonflikt besteht.

Dieser Beitrag beinhaltet keine Studien an Menschen oderTieren.

The supplement containing this article is not sponsored by industry.

\section{Literatur}

1. Freed HA, Shields NN (1984) Most frequently overlooked radiographically apparent fractures in a teaching hospital emergency department. Ann Emerg Med 13:900-904
2. Griffith JF, Roebuck DJ, Cheng JC et al (2001) Acute elbow trauma in children: spectrum of injury revealed by MR imaging not apparent on radiographs. AJR Am J Roentgenol 176:53-60

3. Halsted MJ, Kumar H, Paquin JJ et al (2004) Diagnostic errors by radiology residents in interpreting pediatric radiographs in an emergency setting. Pediatr Radiol 34:331-336

4. Irshad F, Shaw NJ, Gregory RJ (1997) Reliability of fatpad sign in radial head/neck fractures of the elbow. Injury 28:433-435

5. John SD, Wherry K, Swischuk LE, Phillips WA (1996) Improving detection of pediatric elbow fractures by understanding their mechanics. Radiographics 16:1443-1460

6. Kraus R, Berthold LD, Laer L von (2007) Efficient imaging of elbow injuries in children and adolescents. Klin Padiatr 219:282-287

7. Laer L von, Kraus L, Linhart WE (Hrsg) (2007) Frakturen und Luxationen im Wachstumsalter, 5. Aufl. Thieme, Stuttgart New York

8. Marzi I (Hrsg) (2010) Kindertraumatologie, 2. Aufl. Springer, Berlin Heidelberg New York

9. Pistor G, Graffstädt H (2003) Sonographic diagnosis of supracondylar fractures of the humerus. Ultraschall Med 24:331-339

10. Pudas T, Hurme T, Mattila K, Svedström E (2005) Magnetic resonance imaging in pediatric elbow fractures. Acta Radiol 46(6):636-644

11. Rivara FP, Parish RA, Mueller BA (1986) Extremity injuries in children: predictive value of clinical findings. Pediatrics 78:803-807

12. Schneidmueller D, Maier M, Mack M et al (2005) Therapeutic relevance of magnetic resonance imaging in joint injuries in children. Unfallchirurg 108:537-543

13. Simanovsky N, Lamdan R, Mosheiff R, Simanovsky N (2007) Underreduced supracondylar fracture of the humerus in children: clinical significance at skeletal maturity. J Pediatr Orthop 27(7):733-738

14. Skaggs D, Pershad J (1997) Pediatric elbow trauma. Pediatr Emerg Care 13(6):425-434

15. Skaggs DL, Mirzayan R (1999) The posterior fat pad sign in association with occult fracture of the elbow in children. J Bone Joint Surg Am 81:1429-1433

16. Vocke-Hell AK, Schmid A (2001) Sonographic differentiation of stable and unstable lateral condyle fractures of the humerus in children. J Pediatr Orthop B 10:138-141

17. Weinberg AM, Schneidmueller D (Hrsg) (2010) Unfallchirurgie bei Kindern. Deutscher Ärzteverlag, Köln 